VOL. 31 (1985), 235-243.

\title{
ON SUBNORMALITY OF GENERALIZED DERIVATIONS AND TENSOR PRODUCTS
}

\author{
Bojan Magajna
}

\begin{abstract}
Subnormal and quasinormal tensor product operators and generalized derivations on the Hilbert-Schmidt class will be characterized.
\end{abstract}

\section{Introduction}

Let $H$ be a complex Hilbert space, $B(H)$ the algebra of all bounded linear operators on $H$. For $l \leq p<\infty$ the von Neumann-Schatten class, $C_{p}(H)$, is defined to be the set of all elements $T$ in $B(H)$ such that $\sum_{k \in K}\left|\left\langle T \psi_{k}, \psi_{k}\right\rangle\right|^{p}<\infty$ for each orthonormal system $\left\{\psi_{k}: k \in K\right\}$ in $H$ (see [9]). For fixed $A, B \in B(H)$ let $\delta_{A, B}$ and $\tau_{A, B}$ be the operators on $B(H)$ defined by

$$
\begin{aligned}
& \delta_{A, B}(X)=A X-X B, \\
& \tau_{A, B}(X)=A X B .
\end{aligned}
$$

Operators of the form (1) are called generalized derivations and they (as well as their restrictions $\left.\delta_{A, B} \mid C_{p}\right)$ have been extensively studied in the past, especially their spectral properties (see, for example, [8], p, 79

Received 28 September 1984.

Copyright Clearance Centre, Inc. Serial-fee code: 0004-9727/85 $\$ A 2.00+0.00$. 
for some historical notes). In [1] Anderson and Foias obtained the characterization of spectral generalized derivations and Shaw characterized in [10] Hermitian and normal operators of the form $\delta_{A, B} \mid x$ where $X$ is a subspace of $B(H)$ which satisfies suitable conditions (in particular $X$ can be $\left.C_{p}(H)\right)$. Now $C_{2}(H)$ is a Hilbert space with respect to the inner product

$$
\langle X, Y\rangle=\operatorname{tr}\left(Y^{*} X\right), X, Y \in \mathcal{C}_{2}(H)
$$

(where tr denotes the trace) and so the concepts of subnormality and quasinormality make sense. It is a purpose of this note to characterize subnormal and quasinormal operators of type $\delta_{A, B} \mid \mathcal{C}_{2}(H)$ and $\tau_{A, B} \mid C_{2}(H)$.

Note that $\tau_{A, B} \mid C_{2}(H)$ can be identified with $A \otimes B^{*}$ (see [7]) and thus we will obtain in this way a characterisation of subnormal and quasinormal tensor products.

Since the Hilbert space $H$ and the operators $A, B$ will be fixed in what follows, we shall denote simply $C_{2}=C_{2}(H), \delta=\delta_{A, B} \mid C_{2}$, $\tau=\tau_{A, B} \mid C_{2}$.

\section{Subnormality}

By (a special case of) Theorem 2.2 in [10], $\delta$ is normal if and only if $A$ and $B$ are normal operators. The following theorem characterizes subnormal operators $\delta$ and $\tau$. Recall that an operator $S \in B(H)$ is subnormal if and only if there exists a bounded normal operator $N$ on some larger Hilbert space $K \supset H$ such that the restriction of $N$ to $H$ is $S . N$ is then called the normal extension of $S$.

THEOREM 1. Let $\delta$ and $\tau$ be defined on $C_{2}$ by (1) and (2). Then $\delta$ is subnormal if and only if $A$ and $B^{*}$ are subnormal operators. Moreover, if $A \neq 0$ and $B \neq 0$ the same statement holds for $\tau$.

Proof. Suppose first that $A$ and $B^{*}$ are subnormal and denote by $M$ and $N^{*}$ their (not necessarily minimal) normal extensions. Clearly we may assume that $M$ and $N$ act on the same Hilbert space $K \supset H$. Relative to the decomposition $K=H \oplus H^{\perp}$ the operators $M$ and $N^{*}$ can be represented by the matrices 


$$
M=\left(\begin{array}{ll}
A & A_{1} \\
0 & A_{2}
\end{array}\right), \quad N^{*}=\left(\begin{array}{cc}
B^{*} & B_{1} \\
0 & B_{2}
\end{array}\right) \text {, }
$$

where $A_{1}, A_{2}, B_{1}, B_{2}$ are certain bounded operators. Now we can regard $C_{2}=C_{2}(H)$ as a subspace of $C_{2}(K)$ via the embedding

$$
x \mapsto\left(\begin{array}{ll}
x & 0 \\
0 & 0
\end{array}\right), \quad x \in \mathcal{C}_{2}
$$

A straightforward computation with matrices (4) shows that $C_{2}$ is an invariant subspace for the operator $\delta_{M, N}$ defined on $C_{2}(K)$ by $\delta_{M, N}(X)=M X-X N$ and that $\delta_{M, N} \mid C_{2}=\delta$. By Theorem 2.2 of $[10], \delta_{M, N}$ is a normal operator on $C_{2}(K)$ (this can be also verified directly using (3)). Thus $\delta$ is subnormal. The proof that $\tau$ is subnormal is the same since an easy computation gives that the operator $\tau_{M, N}$ is normal on $c_{2}(K)$

To prove the converse we shall use the following theorem of Halmos and Bram (see [2] or [4]).

An operator $T \in B(H)$ is subnormal if and only if

$$
\sum_{j, k=0}^{n}\left\langle T^{j} f_{k}, T^{k} f_{j}\right\rangle \geq 0
$$

for every finite subset $f_{0}, \ldots, f_{n}$ of $H$.

Suppose that $\delta$ is subnormal. In order to apply (5) with $\delta$ instead of $T$ express the powers $\delta^{j}$ by

$$
\delta^{j} X=\sum_{s=0}^{j}(-1)^{s}\left(\begin{array}{l}
j \\
s
\end{array}\right) A^{j-s} X B^{s}, \quad X \in C_{2} .
$$

Taking into account also the definition (3) of inner product in $\mathcal{C}_{2}$ we see that (5) assumes the form

$$
\sum_{j, k=0}^{n} \sum_{r=0}^{j} \sum_{s=0}^{k}(-1)^{r+s}\left(\begin{array}{l}
j \\
r
\end{array}\right)\left(\begin{array}{l}
k \\
s
\end{array}\right) \operatorname{tr}\left(B^{*^{s}} X_{j}^{\star} A^{\star^{k-s}} A^{j-r} X_{k} B^{r}\right) \geq 0
$$


where $x_{1}, \ldots, x_{n}$ are arbitrary elements of $c_{2}$. Now let $f_{j}, g_{j}$, $j=1, \ldots, n$ be any vectors in $H$ and put $x_{j}=f_{j} \otimes g_{j}$ (that is, $\left.X_{j}(h)=\left\langle h, g_{j}\right\rangle f_{j}, h \in H\right\rangle$. Then, after a simple computation, we get, from (6),

$$
\sum_{j, k=0}^{n} \sum_{r=0}^{j} \sum_{s=0}^{k}(-1)^{r+s}\left(\begin{array}{l}
j \\
r
\end{array}\right)\left(\begin{array}{l}
k \\
s
\end{array}\right)\left\langle A^{j-r} f_{k}, A^{k-s} f_{j} \chi^{*^{*} s} g_{j}, B^{*^{r}} g_{k}\right\rangle \geq 0 .
$$

We will show how (7) implies that $A$ is subnormal. The proof that $B^{*}$ is subnormal is similar and will be omitted. Without loss of generality we may assume that 0 is an approximate eigenvalue of $B^{*}$. (Otherwise we can replace $A$ and $B$ with $A-\alpha$ and $B-\alpha$ respectively, where $\bar{\alpha}$ is an approximate eigenvalue for $B^{*}$; this is possible since $\left.\delta_{A, B}=\delta_{A-\alpha, B-\alpha}.\right)$ Let $\left(h_{m}\right)$ be the corresponding sequence of approximate eigenvectors (that is, $\left\|h_{m}\right\|=1$ and $\lim \left\|B^{*} h_{m}\right\|=0$ ). For fixed $m$ put $g_{1}=g_{2}=\ldots=g_{n}=h_{m}$ in $(7)$, then let $m$ tend to infinity. It follows that

$$
\sum_{j, k=0}^{n}\left\langle A^{j} f_{k}, A^{k} f_{j}\right\rangle \geq 0
$$

and this implies that $A$ is subnormal by the Bram-Halmos theorem.

The proof that subnormality of $\tau$ implies subnormality of $A$ and $B^{*}$ is similar. Instead of (7) we have here an analogous condition (derived in the same way as (7))

$$
\sum_{j, k=0}^{n}\left\langle A^{j} f_{k}, A^{k} f_{j} \chi_{B^{*}} g_{j}, B^{* j} g_{k}\right\rangle \geq 0 .
$$

Since $A \neq 0, B \neq 0$ by assumption it follows that $\tau \neq 0$ and hence $\sigma(\tau) \neq\{0\}$ by subnormality. Now the theorem of Brown and Pearcy in [3] tells that $\sigma(\tau)=\sigma(A) \cdot \sigma(B)$, hence there is a $\beta \neq 0$ in the boundary of $\sigma\left(B^{*}\right)$. Then $\beta$ is an approximate eigenvalue of $B^{*} ;$ let $\left(h_{m}\right)$ be the corresponding sequence of eigenvectors. Replace now in (8) all $g_{j}$, $j=1, \ldots, n$, with the same vector $h_{m}$ and then take the limit as $m$ tends to infinity. It follows 


$$
\sum_{j, k=0}^{n} B^{k-j}\left\langle A^{j} f_{k}, A^{k} f_{j}\right\rangle \geq 0
$$

and this implies that $A$ is subnormal since $f_{j}$ are arbitrary and $B \neq 0$. The proof that $B^{*}$ is subnormal is similar. //

\section{Quasinormality}

An operator $T \in B(H)$ is called quasinormal if and only if it commutes with $T^{*} T$ ([4], [6]).

THEOREM 2. Let $\delta$ and $\tau$ be defined on $c_{2}$ by (1) and (2).

(i) $\delta$ is quasinormal if and only if one of the following holds:

(a) $A$ and $B$ are both normal;

(b) there exists $\lambda \in \mathbb{C}$ such that $A=\lambda I$ and $(B-\lambda I)^{*}$ is quasinormal;

(c) there exists $\lambda \in \mathbb{C}$ such that $B=\lambda I$ and $A-\lambda I$ is quasinormal.

Here of course $I$ is the identity operator on $H$.

(ii) If $A \neq 0$ and $B \neq 0$ then $\tau$ is quasinormal if and only if $A$ and $B^{*}$ are quasinormal.

In the proof of this theorem the following result of Fong and Sourour will be used (see [5]).

(FS) Let $\mathrm{A}=\left\{A_{1}, \ldots, A_{n}\right\}$ and $B=\left\{B_{1}, \ldots, B_{n}\right\}$ be finite subsets of $B(H)$. Suppose that

$$
A_{1} X B_{1}+\ldots+A_{n} X B_{n}=0
$$

for all $X \in C_{2}$ and that $A_{1}, \ldots, A_{k}$ are linearly independent. Then $B_{1}, \ldots, B_{k}$ can be expressed as linear combinations of $B_{k+1}, \ldots, B_{n}$. (In particular for $k=n$ this means $B_{1}=\ldots=B_{n}=0$. Also the role of $A$ and $B$ can be interchanged.)

Actually in [5] this result is stated for $B(X)$ (where $X$ is any Banach space) instead of $\mathcal{C}_{2}$ but (FS) follows at once since $\mathcal{C}_{2}$ is 
strongly dense in $B(H)$.

Proof of Theorem 2. (i) Since $\delta^{*}$, the adjoint of $\delta$, is given by $\delta^{*}(X)=A^{*} X-X B^{*}$ (as a direct verification would show) the quasinormality condition $\delta^{*} \delta^{2}-\delta \delta * \delta=0$ can be written as

$$
\begin{array}{r}
\left(A^{*} A^{2}-A A^{*} A\right) X I+\left(A A^{*}-A^{*} A\right) X B-A X\left(B^{*} B-B B^{*}\right)-X\left(B^{2} B^{*}-B B^{*} B\right)=0, \\
\text { for all } X \in \mathcal{C}_{2},
\end{array}
$$

where $I$ denotes the identity operator.

If $B$ is not normal then $I$ and $B^{*} B-B B^{*}$ are linearly independent since 0 is the only scalar commutator ([6], Problem 230). Hence it follows from (9) by (FS) that $A$ can be expressed as a linear combination of commuting self-adjoint operators $I$ and $A A^{*}-A^{*} A$. Thus $A$ is normal and in fact a scalar multiple of $I$. If we put $A=\lambda I$ in (9) we get

$$
B^{2} B^{*}-B B^{*} B+\lambda\left(B^{*} B-B B^{*}\right)=0
$$

This equation can be written also as

$$
(B-\lambda I)^{2}(B-\lambda I)^{*}-(B-\lambda I)(B-\lambda I) *(B-\lambda I)=0
$$

which is obviously equivalent to the quasinormality of $(B-\lambda I) *$.

The case when $A$ is not normal is treated in the same way. Now only the case when $A$ and $B$ are both normal remains, but then $\delta$ is normal.

(ii) Since $\tau^{*}(X)=A^{*} X B^{*}, X \in \mathcal{C}_{2}$, the quasinormality condition $\tau * \tau^{2}-\tau \tau^{*} \tau=0$ is equivalent to

$$
A A^{*} A X B B^{*} B-A^{*} A^{2} X B^{2} B^{*}=0, \quad X \in C_{2} .
$$

If $A$ and $B^{*}$ are quasinormal then obviously (10) is satisfied.

Conversely, if (10) is satisfied then $A A^{*} A$ and $A^{*} A^{2}$ are linearly dependent. (Otherwise it would follow that $B B^{*} B=0$ by (FS) and hence $\|B\|^{4}=\left\|B^{*} B B^{*} B\right\|=0$, but $B \neq 0$ by assumption.) Thus we have

$$
A^{*} A^{2}=\lambda A A^{*} A
$$

for some $\lambda \in \mathbf{C}$. If we prove that $\lambda=1$ then $A$ will be quasinormal 
and since the quasinormality of $B^{*}$ can be proved similarly this will complete the proof of the theorem. Now (11) implies that

$$
A^{*} A^{2}=\lambda A^{*} A A^{\star} A
$$

Since $A^{*} A^{2}$ and $A^{*} A A^{*} A$ are non-negative operators different from 0 , (12) implies that $\lambda \geq 0$. From Theorem $I$ and the fact that every quasinormal operator is subnormal ([6], Problem 195) we see that $A$ is subnormal, hence $\left\|A^{2}\right\|=\|A\|^{2}$. From comparing the norms of the left and the right side of (12) it follows that $\lambda=1 . / /$

\section{Hyponormality}

An operator $T \in B(H)$ is hyponormal (by definition) if and only if $T^{*} T-T T^{*} \geq 0$.

If $A$ and $B^{*}$ are hyponormal operators then $\delta$ is also hyponormal by [10], p. 141. Actually the argument of [10] together with the fact that 0 is always in the closure of the numerical range of $A^{*} A-A A^{*}$ (where $A \in B(H)$ ) imply that the converse is also true. A similar statement can be proved for $\tau$.

PROPOSITION. Suppose $A \neq 0, B \neq 0$. Then $\tau$ is hyponormal if and only if $A$ and $B^{*}$ are hyponormal.

Proof. Note first that the hyponormality condition for $\tau$,

$$
0 \leq\left(\left(\tau^{*} \tau-\tau \tau^{*}\right) X, X\right)=\operatorname{tr}\left(X^{*}\left(A^{*} A X B B^{*}-A A^{*} X B^{*} B\right)\right), \quad X \in C_{2},
$$

can be written in the form

$$
\operatorname{tr}\left(B^{*} X^{*}\left(A^{*} A-A A^{*}\right) X B\right)+\operatorname{tr}\left(A^{*} X\left(B B^{*}-B^{*} B\right) X^{*} A\right) \geq 0, \quad X \in \mathcal{C}_{2} .
$$

(Here we have used the identity $\operatorname{tr}(Y Z)=\operatorname{tr}(Z Y)$ for $Y \in B(H)$, $Z \in C_{1}(H)$ and for $Y, Z \in C_{2}(H)\left([9]\right.$, p. 100).) If $A$ and $B^{*}$ are hyponormal then $(X B)^{*}\left(A^{*} A-A A^{*}\right) X B \geq 0$ and $\left(X^{*} A\right)^{*}\left(B B^{*}-B^{*} B\right) X^{*} A \geq 0$ for all $X \in \mathcal{C}_{2}$ and so (14) holds.

Conversely, if $\tau$ is hyponormal then put $X=f \otimes g$ in (13) where $f, g \in H$. It follows, after a short computation,

$$
\|A f\|\left\|B^{*} g\right\|-\left\|A^{*} f\right\|\|B g\| \geq 0 \text {. }
$$


We shall prove that $B^{*}$ is hyponormal, the proof that $A$ is hyponormal is similar. Assume for a moment that there exists a sequence $\left(f_{m}\right)$ of unit vectors in $H$ such that $\lim \left\|A^{*} f_{m}\right\|=\lim \left\|A f_{m}\right\|>0$. Then the hyponormality of $B^{*}$ follows at once from (15) if we put $f=f_{m}$ and take the limit when $m$ tends to infinity. To prove that the sequence $\left(f_{m}\right)$ exists put $C=A^{*} A-A A^{*}$. If $C \geq 0$ (respectively $C \leq 0$ ) let $\alpha$ be any nonzero approximate eigenvalue for $A$ (respectively $A^{*}$ ); then the corresponding sequence of unit approximate eigenvectors satisfies the requirement. (Proof. The relations $\lim (A-\alpha) f_{n}=0$ and $(A-\alpha)^{*}(A-\alpha)-(A-\alpha)(A-\alpha) *=C \geq 0$ imply $\lim (A-\alpha)^{*} f_{n}=0$, thus $\left.\lim \left\|A^{*} f_{m}\right\|=|\alpha|=\lim \left\|A f_{m}\right\|.\right)$ If neither $c \geq 0$ nor $c \leq 0$ then there exists $f \in H,\|f\|=1$, such that $(C f, f)=0$ and $\|C f\| \neq 0$. (This can be seen from the spectral theorem when $C$ is represented as a multiplication with a bounded measurable real function on a suitable $L^{2}(\mu)$.) Now the constant sequence, $f_{m}=f$, satisfies the requirement. //

Let us finally remark that the same kind of characterization can not hold for general elementary operators. For example the operator $X \mapsto A X B+A^{*} X B^{*}$ is self-adjoint on $C_{2}$ for arbitrary $A, B \in B(H)$.

\section{References}

[1] J. Anderson and C. Foias, "Properties which normal operators share with normal derivations and related operators", Pacific J. Math. 61 (1975), 313-325.

[2] J. Bram, "Subnormal operators", Duke Math. J. 22 (1955), 75-94.

[3] A. Brown and C.M. Pearcy, "Spectra of tensor product of operators", Proc. Amer. Math. Soc. 17 (1966), 162-169.

[4] J.B. Conway, Subnormal operators (Pitman, Boston, 1981).

[5] C.K. Fong and A.R. Sourour, "On the operator identity $\sum A_{k} X B_{k}=0$ ", Canad. J. Math. 31 (1979), 845-857. 
[6] Paul R. Halmos, A Hilbert space problem book (Springer-Verlag, New York, Heidelberg, Berlin, 1982).

[7] Paul R. Halmos and V.S. Sunder, Bounded integral operators on $L^{2}$ spaces (Springer-Verlag, Berlin, Heidelberg, New York, 1978).

[8] D.A. Herrero, Approximation of Hilbert space operators, Volume I (Pitman, Boston, 1982).

[9] J.R. Ringrose, Compact non-self-adjoint operators (Van Nostrand, London, 1971).

[10] S.Y. Shaw, "On numerical ranges of generalized derivations and related properties", J. Austral. Math. Soc. Ser. A 36 (1984), $134-142$.

Department of Mathematics,

University of Ljubljana,

Jadranska 19,

Ljubljana 61000,

Yugoslavia. 